defined as an extended response time in the delivery of airway pressure following initiation of inspiratory effort by the patient. Although bench tests of ventilators have demonstrated variation in trigger response times $(120-500 \mathrm{~ms})$, there are limited data reporting the effect of trigger delay on a subject's NRD and comfort. We therefore investigated the relationship between ventilator trigger delay, NRD and comfort perception.

Methods A custom-made NIPPY3 + ventilator (B\&D Electromedical, Stratford-upon-Avon, UK) with modifiable trigger delay was used. A standardised protocol of $10 \mathrm{~cm} \mathrm{H} 20$ inspiratory positive airway pressure, $4 \mathrm{~cm} \mathrm{H} 20$ expiratory positive airway pressure and back up rate of 6 breaths per minute was utilised in healthy subjects familiarised with NIV. Subjects were blinded to the settings and asked to assess perceived comfort using a visual analogue score (VAS) at 20 randomised trigger delay timings ranging from $10 \mathrm{~ms}$ to $1000 \mathrm{~ms}$ following a 2 minute assessment period. Second intercostal space electromyography (EMGpara\% max), as a marker of NRD, mask pressure and flow were used to assess PVI. Transcutaneous carbon dioxide and oxygen saturations were controlled within limits of $0.5 \mathrm{kPa}$ and $4 \%$ respectively of the subject's baseline values to minimise changes in the biochemical drive to breathe.

Results 5 subjects ( 1 male) were enrolled to date with a mean age of $34 \pm$ 8years, BMI $22 \pm 3 \mathrm{kgm}^{2}, \mathrm{FEV}_{1} 105 \pm 11 \%$ predicted and FVC $114 \pm 13 \%$ predicted. 500 breaths were analysed. The EMGpara\%max was lowest at a trigger delay setting of $400 \mathrm{~ms} 5.9 \%(4.8-8.0)$ and largest at a trigger delay of $800 \mathrm{~ms}$ 10.1\%(6.0-16.5). (Figure 1). There was a corresponding decrease in the VAS score from $78 \mathrm{~cm}(63.5-92.5)$ at $400 \mathrm{~ms}$
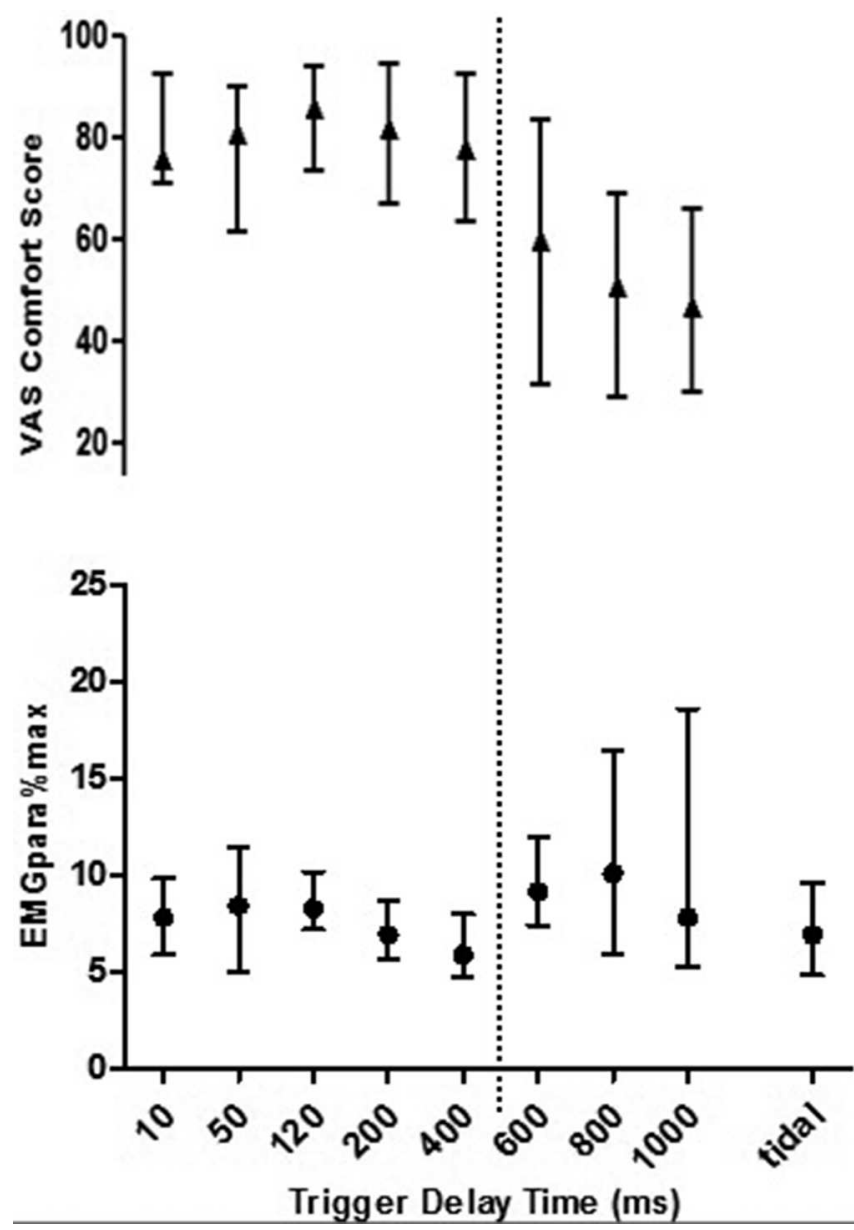

Abstract S87 Figure 1. Graph representing the changes in comfort and neural respiratory drive with increasing trigger delay trigger delay to $47 \mathrm{~cm}(30-66)$ at $1000 \mathrm{~ms}$ trigger delay. The highest comfort score was $89 \mathrm{~cm}(52-92)$ observed at $170 \mathrm{~ms}$ trigger delay.

Conclusion This is the first study to comprehensively investigate NIV trigger delay in healthy subjects. Based on NRD, these data suggest that the optimal NIV trigger response time was up to $400 \mathrm{~ms}$. This challenges previous bench studies that reported ventilators with response times over $100-150 \mathrm{~ms}$ have limited clinical utility.

\section{S88 1 YEAR UK SURVIVAL READMISSION RATE IN CHRONIC OBSTRUCTIVE PULMONARY DISEASE (COPD) SURVIVORS FOLLOWING ACUTE NON-INVASIVE VENTILATION (NIV)}

${ }^{1} \mathrm{~S}$ Keilty, ${ }^{2} \mathrm{P}$ Murphy, ${ }^{3 \mathrm{P}}$ Murphy, ${ }^{2} \mathrm{~N}$ Hart, ${ }^{3} \mathrm{~N}$ Hart, ${ }^{4} \mathrm{~N}$ Hart; ${ }^{1}$ Department of Physiotherapy, Guy's \& St Thomas' NHS Foundation Trust, London, UK; ${ }^{2}$ Lane Fox Clinical Respiratory Physiology Research Centre, Guy's \& St Thomas' NHS Foundation Trust, London, UK; ${ }^{3}$ Division of Asthma, Allergy and Lung Biology, King's College, London, UK; ${ }^{4}$ Lane Fox Respiratory Unit, Guy's \& St Thomas' NHS Foundation Trust, London, UK

\subsection{6/thoraxjn-2013-204457.95}

Background Previous data has shown that premorbid MRCdyspnoea (MRCD) score predicts outcomes following acute exacerbation of COPD (Steer, Thorax 2012). There are limited data on the clinical utility of the MRCD in predicting outcomes in patients requiring NIV to treat hypercapnic respiratory failure complicating an acute exacerbation.

Methods A single centre observational cohort study was undertaken from $1^{\text {st }}$ April 2009 to $31^{\text {st }}$ March 2012. We prospectively followed a cohort of patients admitted with acute exacerbation requiring NIV according to standard BTS criteria. Pre-admission MRCD were calculated from a prospective database obtained from the electronic patient record system as were hospital readmissions and mortality data.

Results 119 patients (69 male) with a mean age of $71 \pm 12$ years were admitted requiring NIV. Mean $\mathrm{pH}$ was $7.26 \pm 0.07$ with a $\mathrm{PaCO}_{2} 9.47 \pm 2.26 \mathrm{kPa}$ and $\mathrm{HCO}_{3^{-}}$of $30.6 \pm 6.3$ mmols/l. 102 patients (86\%) survived the index hospital admission. $16(16 \%)$ patients had previous admissions requiring acute NIV prior to April 2009. 63 (62\%) survivors were readmitted or died within 1 year with 26 (25\%) survivors having $>1$ readmission episode and 33 (32\%) patients required NIV on readmission within a year. Median 1 year re-admission rate per patient was 1 (1-9) with median time to readmission 228 days for all patients and 373 days, 216 days, 105 days for MRC-dyspnoea score 23,4 and 5, respectively. Median length of stay of the index admission was 9 days (1-48) with median length of readmission, or readmissions of 13 days (1-111). Hospital readmission accounted for 1251 bed days. There was significantly poorer 1 year survival in the most severely dyspnoeic patients (MRC 5)

Kaplan-Meier plot: 1 year survival stratified by MRC dyspnoea score

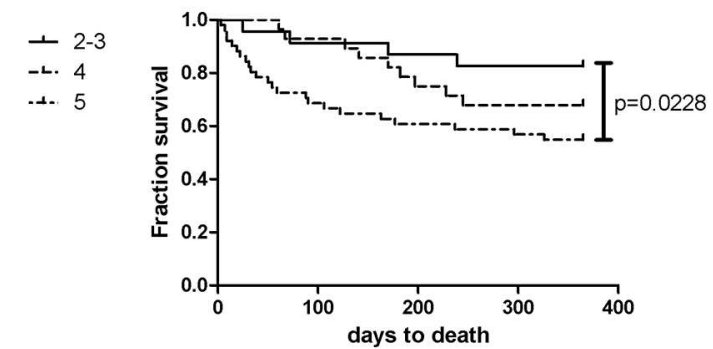

Abstract S88 Figure 1. 
compared to the least (MRC 2 and 3) (HR 0.40, 95\% CI 0.18$0.88, \mathrm{p}=0.023$ ).

Conclusion These data show that COPD patients who receive acute NIV have high risk of hospital readmission including requirement for repeat NIV treatment, which contributed to a significant number of hospital bed days. Although overall outcomes are better than previously reported (Murray, Thorax 2011), patients with high levels of premorbid dyspnoea have the highest mortality following acute hypercapnic exacerbations of COPD requiring NIV.

\section{Airways disease: fungus and the bogeyman}

\section{S89 THE USE OF ASPERGILLUS POLYMERASE CHAIN REACTION TESTING TO GAIN A FURTHER UNDERSTANDING OF SEVERE FUNGAL ALLERGIC ASTHMA}

JP Farrant, L Holmes, R Masania, L Chishimba, R Niven; University Hospital of South Manchester, Manchester, United Kingdom

\subsection{6/thoraxjnl-2013-204457.96}

Background Severe Asthma with Fungal Sensitisation (SAFS) is a newly described phenotype of Fungal Allergic Asthma. There is much debate about the disease's mechanism of action, the best method of treatment and how treatment with antifungal agents such as Itraconazole brings about the improvement in disease control that has been shown in previous studies.

This study aims to answer those questions, through the use of Sputum Aspergillus Polymerase Chain Reaction testing as a method of determining whether a patient has pulmonary colonisation with Aspergillus.

Methods PCR samples were collected between September 2012 and May 2013 samples taken clinically in the previous 2 years were also included. Patients had their antifungal therapy status recorded and received Total serum IgE and Aspergillus Specific IgE testing when providing sputum samples. The study was split into 2 arms. The primary retrospective opportunistic arm had a patient cohort of 135 who provided 254 samples for testing and analysis. The secondary prospective arm of the study looked at 10 patient's commencing Itraconazole therapy. Patients received PCR testing before commencing treatment and then at every opportunity whilst on treatment.

Results

\begin{tabular}{llll}
\hline & PCR Positives & PCR Negatives & $X^{2}$ (p value) \\
SAFS Patients Off & $61(70 \%)$ & $26(30 \%)$ & $37.90(<0.0001)$ \\
$\begin{array}{l}\text { Antifungal Treatement } \\
\text { Control }\end{array}$ & $3(9 \%)$ & $32(91 \%)$ & \\
\hline
\end{tabular}

The primary study arm showed a 70\% rate of pulmonary colonisation in the Untreated Severe Asthma population, which differs significantly to the $9 \%$ rate of positivity seen in the control population. The rate of PCR positivity fell to $23 \%$ in the SAFS population who were receiving treatment. The secondary arm showed that Itraconazole removed fungus from the airways of 9 patients; this was correlated with a decrease in patient's total serum IgE's.

Discussion The $70 \%$ rate of PCR positivity in the untreated SAFS population supports the concept that patients with SAFS have pulmonary colonisation with Aspergillus. The study has also shown that the antifungal agent Itraconazole removes this fungus from patient's airways and that is correlated with an improvement in patient's disease control. This study supports the use of Itraconazole in patients with SAFS.

\section{S90 EFFECTIVENESS OF VORICONAZOLE IN THE TREATMENT OF ASPERGILLUS FUMIGATUS ASSOCIATED ASTHMA}

J Agbetile, M Bourne, A Fairs, B Hargadon, D Desai, C Broad, J Morley, P Bradding, C Brightling, R Green, P Haldar, C Pashley, I Pavord, A Wardlaw; University of Leicester, Department of 3I's, Leicester, United Kingdom

\subsection{6/thoraxjnl-2013-204457.97}

Background IgE sensitisation to Aspergillus fumigatus and a positive sputum fungal culture are common in refractory asthma. It is not clear whether these patients would benefit from anti-fungal treatment.

Objectives To determine if a three-month course of voriconazole improved asthma related outcomes in people with asthma who are $\operatorname{IgE}$ sensitised to A. fumigatus.

Methods Asthmatics IgE sensitised to A fumigatus with a history of at least two severe exacerbations in the previous twelve months were treated for three months with voriconazole two hundred milligrams twice daily, followed by observation for nine months, in a double blind, placebo controlled, randomised design. Primary outcomes were improvement in quality of life at the end of the treatment period and a reduction in the number of severe exacerbations over the twelve months of the study.

Results 65 patients were randomised. 59 patients started treatment (32 voriconazole and 27 placebo) and were included in an intention to treat analysis. 56 patients took the full three months of medication. There was no significant difference in the number of severe exacerbations between the voriconazole and placebo groups (1.25 vs 1.52/patient/year; mean difference 0.27 ; $95 \%$ CI 0.24 to 0.31 ) respectively, quality of life (change in AQLQ 0.44 vs 0.35 , mean difference between groups 0.08 ; 95\% CI $0.07-$ 0.09 ), or in any of our secondary outcome measures between the two groups.

Conclusion We were unable to show a beneficial effect of three months treatment with voriconazole in people with moderate to severe asthma who were IgE sensitised to A fumigatus on either the rate of severe exacerbations, quality of life or other markers of asthma control.

\section{REFERENCES}

1. Fairs $A$, Agbetile J, Hargadon B, Bourne $M$, Monteiro WR, Brightling $C E$, et al. IgE sensitisation to Aspergillus fumigatus is associated with reduced lung function in asthma. Am J Respir Crit Care Med. 2010;182(11):1362-8.

Juniper Asthma quality of Life

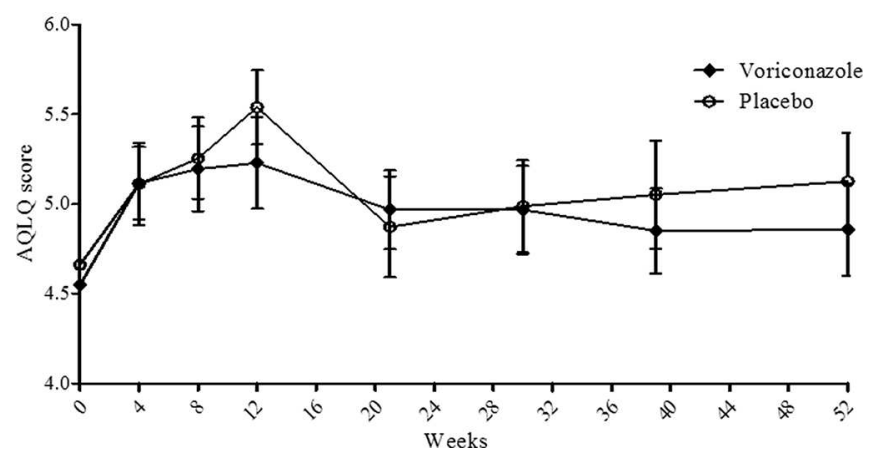

Abstract S90 Figure 1. 